\title{
MCNPX simulations of the silicon carbide semiconductor detector response to fast neutrons from $D-T$ nuclear reaction
}

\author{
Katarína Sedlačková \\ Institute of Nuclear and Physical Engineering, Slovak University of Technology, Ilkovičova 3, \\ 81219 Bratislava, Slovakia \\ katarina.sedlackova@stuba.sk \\ Andrea Šagátová \\ University Centre of Electron Accelerators, Slovak Medical University in Bratislava, Ku kyselke 497, \\ 91106 Trenčin, Slovakia \\ andrea.sagatova@stuba.sk \\ Bohumír Zat'ko \\ Institute of Electrical Engineering, Slovak Academy of Sciences, Dúbravská cesta 9, \\ 84104 Bratislava, Slovakia \\ elekbzat@savba.sk \\ Vladimír Nečas \\ Institute of Nuclear and Physical Engineering, Slovak University of Technology, Ilkovičova 3, \\ 81219 Bratislava, Slovakia \\ vladimir.necas@stuba.sk \\ Michael Solar* and Carlos Granja ${ }^{\dagger}$ \\ Institute of Experimental and Applied Physics, Czech Technical University in Prague, Horská 3a/22, \\ CZ 12800 Prague 2, Czech Republic \\ *michael.solar@cvut.utef.cz, ${ }^{\dagger}$ carlos.granja@utef.cvut.cz \\ Published 1 September 2016
}

\begin{abstract}
Silicon Carbide (SiC) has been long recognized as a suitable semiconductor material for use in nuclear radiation detectors of high-energy charged particles, gamma rays, X-rays and neutrons. The nuclear interactions occurring in the semiconductor are complex and can be quantified using a Monte Carlo-based computer code. In this work, the MCNPX (Monte Carlo N-Particle eXtended) code was employed to support detector design and analysis. MCNPX is widely used to simulate interaction of radiation with matter and supports the transport of 34 particle types including heavy ions in broad energy ranges. The code also supports complex 3D geometries and both nuclear data tables and physics models. In our model, monoenergetic neutrons from D-T nuclear reaction were assumed as a source of fast neutrons. Their energy varied between 16 and $18.2 \mathrm{MeV}$, according to the accelerating voltage of the deuterons participating in $\mathrm{D}-\mathrm{T}$ reaction. First, the simulations were used to calculate the optimum thickness of the reactive film composed of High Density PolyEthylene (HDPE), which converts neutral particles to charged particles and thusly enhancing detection efficiency. The dependency of the optimal thickness of the HDPE layer on the energy of
\end{abstract}

This is an Open Access article published by World Scientific Publishing Company. It is distributed under the terms of the Creative Commons Attribution 3.0 (CC-BY) License. Further distribution of this work is permitted, provided the original work is properly cited. 


\title{
K. Sedlačková et al.
}

\begin{abstract}
the incident neutrons has been shown for the inspected energy range. Further, from the energy deposited by secondary charged particles and recoiled ions, the detector response was modeled and the effect of the conversion layer on detector response was demonstrated. The results from the simulations were compared with experimental data obtained for a detector covered by a 600 and $1300 \mu \mathrm{m}$ thick conversion layer. Some limitations of the simulations using MCNPX code are also discussed.
\end{abstract}

Keywords: Radiation detectors; Monte Carlo simulations.

PACS numbers: 29.40.-n, 24.10.Lx.

\section{Introduction}

Silicon carbide $(\mathrm{SiC})$ is a prospective compound semiconductor material for fabrication of radiation detectors aimed to operate in harsh environments. Its wide bandgap $(3.26 \mathrm{eV}$ for the $4 \mathrm{H}-\mathrm{SiC}$ polytype) and high displacement energy of both $\mathrm{Si}$ and $\mathrm{C}$ atoms ( 35 and $20 \mathrm{eV}$, respectively) are particularly interesting properties that offer a significant advantage for high-temperature and high-fluence applications. The potential of $\mathrm{SiC}$ detectors to withstand high temperatures up to $700^{\circ} \mathrm{C}$ and high dose rates when irradiated by electrons, alpha particles, light ions, neutrons and gamma-rays have been reported. ${ }^{1-7}$

$\mathrm{SiC}$ detectors are being developed for high-energy charged particle, UV, X-ray, gamma-ray and neutron spectrometry. The spectroscopic performance of the $\mathrm{SiC}$ detectors based on high purity epitaxial material is, however, limited by the achievable thickness of the depletion region. Present status of the fabrication technology enables production of a good quality material and promotes active development of $\mathrm{SiC}$ detectors.

In this paper, we focused our interest on $\mathrm{SiC}$ detectors for fast neutrons. Detection of neutrons is limited regarding their spectrometry. In spite of a possibility to detect fast neutrons using a bare $\mathrm{SiC}$ material, a reactive material is usually essential to improve its relatively low detection efficiency. Thus, the particle transport through more media becomes more complicated and can be favorably modeled using a Monte-Carlo computer code. MCNPX (Monte Carlo N-Particle eXtended) ${ }^{8}$ code is a good tool to contribute to detector design and its optimization. We have shown its possibilities to optimize a reactive layer for a detector registering radiation from ${ }^{239} \mathrm{PuBe}$, a radioisotope source ${ }^{9}$ with a mixed neutron and gamma yield. Additionally, we present simulations concerning detection of monoenergetic neutrons modeled from D-T nuclear reactions. The thickness of the reactive layer was calculated and its effect on detector response was evaluated. A comparison with experimentally obtained data is also discussed.

\section{Detection of Fast Neutrons by SiC Detector with Conversion Layer}

Fast neutrons can be registered using $\mathrm{SiC}$ material directly as a result of nuclear reactions that produce charged-particle products. The primary reactions causing the detector response are the ${ }^{12} \mathrm{C}\left(\mathrm{n}, \mathrm{n}^{\prime}\right){ }^{12} \mathrm{C}$ and ${ }^{28} \mathrm{Si}\left(\mathrm{n}, \mathrm{n}^{\prime}\right)^{28} \mathrm{Si}$ elastic and inelastic scattering. Due to the lower mass of carbon atom compared to silicon, more neutron kinetic energy can be transferred to carbon recoil than to silicon recoil. Consequently, the recoil continuum ascribed to carbon can be recognized in detector response at higher energies. In addition 
to recoil carbon and silicon ions, charged particle products (alpha particles and protons) from other nuclear reactions contribute a measurable signal when the energy of the registered neutron reaches the threshold for the respective reaction. Because the energy of 16.031 MeV exceeds all reaction thresholds, the fast neutron detector response is very complex. The most prominent fast-neutron-induced reactions are listed in Table 1. The dominant peak in the detector response is usually the ${ }^{12} \mathrm{C}(\mathrm{n}, \alpha){ }^{9} \mathrm{Be}$ reaction on carbon yielding $10.33 \mathrm{MeV}$ shared between the $\alpha$-particle and the ${ }^{9} \mathrm{Be}$ recoil.

Coating the detectors with an appropriate neutron reactive material substantially enhances the registration of neutrons. Neutrons interacting in the reactive coating, i.e. the conversion layer, eject secondary products that can enter the active volume of the detector material and contribute to the output signal. For fast neutrons, the most commonly investigated reactive material is High Density Polyethylene (HDPE) because of its high concentration of hydrogen, which exhibits a high elastic scattering crosssection for fast neutrons. Elastic scattering of fast neutrons impinging hydrogen nuclei results in production of recoil protons that can be easily registered when entering the active volume of a detector. As a result of a variable amount of energy transferred from a neutron to a hydrogen atom, a complex spectrum of proton recoils can be expected even in case of monoenergetic neutron registration. Therefore, the neutron spectrometry of semiconductor detectors with an HDPE layer is also limited.

\section{Method and Calculation Details}

MCNPX code (version 2.7.0) ${ }^{8}$ was used to simulate the experiment of fast neutron registration by a $\mathrm{SiC}$ detector with and without the HDPE conversion layer. The detector was modeled as a cylinder filled with $\mathrm{SiC}$ with a diameter of $1.4 \mathrm{~mm}$. Its thickness varied according to applied reverse bias in real experiments, i.e. 44, 55 and $64 \mu \mathrm{m}$ correspond to an active volume thickness with an applied reverse bias of $-200,-300$ and $-400 \mathrm{~V}$, respectively. A point source of neutrons is incident from a perpendicular distance of $1 \mathrm{~cm}$ to the detector. A Schottky contact plane was assumed in the simulations. The outside medium was air. The source neutron energies were chosen according to neutron energies from the $t(d, n) \alpha$ reaction, which are as follows: for deuteron energies of $E_{d}=0.6,1,1.5$ and $2 \mathrm{MeV}$, the corresponding neutron energies are $\mathrm{E}_{\mathrm{n}}=16.031,16.755,17.543$ and 18.262 MeV, respectively.

We first calculated the thickness of the conversion film yielding the maximum incident neutron-to-recoil-proton conversion efficiency. This was quantified as the ratio of the proton flux density on the back side of the HDPE film to the neutron flux density on the top side of the conversion layer (i.e. F2 tallies in the MCNPX code). The recoil option on the neutron physics card was activated to ensure the transport of the recoil protons. The calculated value of the reactive film thickness is expected to be shorter than the range of the most energetic protons in HDPE. As the amount of energy transferred to the protons varies, a more complex distribution of proton ranges can be expected. 
Table 1. Fast neutron reactions on $\mathrm{SiC}$ and the respective neutron energy thresholds, Q-values and the total recoil energies for $16.031 \mathrm{MeV}$ neutrons. ${ }^{1,10,11}$

\begin{tabular}{|c|c|c|c|}
\hline Reaction & $\begin{array}{l}\text { Neutron energy } \\
\text { threshold [MeV] }\end{array}$ & $\begin{array}{l}Q \text {-value } \\
{[\mathrm{MeV}]}\end{array}$ & $\begin{array}{c}\text { Total charged recoil } \\
\text { energy }[\mathrm{MeV}]\end{array}$ \\
\hline${ }^{28} \mathrm{Si}\left(\mathrm{n}, \mathrm{n}^{\prime}\right){ }^{28} \mathrm{Si}$ ground state & 0 & 0 & continuum \\
\hline${ }^{28} \mathrm{Si}\left(\mathrm{n}, \mathrm{n}^{\prime}\right)^{28 *} \mathrm{Si}\left(1^{\mathrm{st}}\right.$ excited state $)$ & 1.843 & -1.779 & 14.252 \\
\hline${ }^{28} \mathrm{Si}(\mathrm{n}, \alpha){ }^{25} \mathrm{Mg}$ (ground state) & 2.749 & -2.654 & $\alpha_{0} 13.377$ \\
\hline${ }^{28} \mathrm{Si}(\mathrm{n}, \alpha){ }^{25} \mathrm{Mg}\left(1^{\text {st }}\right.$ excited state $)$ & 3.354 & -3.239 & $\alpha_{1} 12.793$ \\
\hline${ }^{28} \operatorname{Si}(\mathrm{n}, \alpha){ }^{25} \mathrm{Mg}\left(2^{\text {nd }}\right.$ excited state $)$ & 3.758 & -3.628 & $\alpha_{2} 12.403$ \\
\hline${ }^{28} \mathrm{Si}(\mathrm{n}, \alpha){ }^{25} \mathrm{Mg}\left(3^{\text {rd }}\right.$ excited state $)$ & 4.418 & -4.266 & $\alpha_{3} 11.766$ \\
\hline${ }^{28} \mathrm{Si}(\mathrm{n}, \alpha){ }^{25} \mathrm{Mg}\left(4^{\text {th }}\right.$ excited state $)$ & 4.783 & -4.618 & $\alpha_{4} 11.413$ \\
\hline${ }^{28} \mathrm{Si}(\mathrm{n}, \alpha){ }^{25} \mathrm{Mg}\left(5^{\text {th }}\right.$ excited state $)$ & 5.650 & -5.455 & $\alpha_{5} 10.576$ \\
\hline${ }^{28} \mathrm{Si}(\mathrm{n}, \alpha){ }^{25} \mathrm{Mg}\left(6^{\text {th }}\right.$ excited state $)$ & 6.275 & -6.059 & $\alpha_{6} 9.972$ \\
\hline${ }^{28} \mathrm{Si}(\mathrm{n}, \mathrm{p}){ }^{28} \mathrm{Al}$ & 3.999 & -3.860 & $\mathrm{p} 12.171$ \\
\hline${ }^{12} \mathrm{C}\left(\mathrm{n}, \mathrm{n}^{\prime}\right){ }^{12} \mathrm{C}$ (ground state) & 0 & 0 & continuum \\
\hline${ }^{12} \mathrm{C}\left(\mathrm{n}, \mathrm{n}^{\prime}\right){ }^{12^{*}} \mathrm{C}\left(1^{\mathrm{st}}\right.$ excited state $)$ & 4.809 & -4.439 & 11.592 \\
\hline${ }^{12} \mathrm{C}\left(\mathrm{n}, \mathrm{n}^{\prime}\right){ }^{12 *} \mathrm{C}\left(2^{\text {nd }}\right.$ excited state $)$ & 8.292 & -7.654 & ${ }^{*}{ }^{1}$ \\
\hline${ }^{12} \mathrm{C}\left(\mathrm{n}, \mathrm{n}^{\prime}\right){ }^{12^{*} \mathrm{C}}\left(3^{\text {rd }}\right.$ excited state $)$ & 11.158 & -9.641 & *1 \\
\hline${ }^{12} \mathrm{C}\left(\mathrm{n}, \mathrm{n}^{\prime}\right){ }^{12^{*}} \mathrm{C}\left(4^{\text {th }}\right.$ excited state $)$ & 13.769 & -10.300 & ${ }^{*} 1$ \\
\hline${ }^{12} \mathrm{C}\left(\mathrm{n}, \mathrm{n}^{\prime}\right) 3 \alpha$ & 7.886 & -7.475 & $7.602^{* 2}$ \\
\hline${ }^{12} \mathrm{C}(\mathrm{n}, \alpha){ }^{9} \mathrm{Be}$ (ground state) & 6.180 & -5.701 & $\alpha_{0} 10.330$ \\
\hline${ }^{12} \mathrm{C}(\mathrm{n}, \alpha){ }^{9} \mathrm{Be}\left(1^{\text {st }}\right.$ excited state $)$ & 8.001 & -7.386 & $\alpha_{1} 8.645^{* 3}$ \\
\hline${ }^{12} \mathrm{C}(\mathrm{n}, \alpha){ }^{9} \mathrm{Be}\left(2^{\text {nd }}\right.$ excited state $)$ & 8.808 & -8.131 & $\alpha_{2} 7.900^{* 3}$ \\
\hline${ }^{12} \mathrm{C}(\mathrm{n}, \mathrm{p}){ }^{12} \mathrm{~B}$ (ground state) & 13.645 & -12.587 & $\mathrm{p}_{0} 3.444$ \\
\hline${ }^{12} \mathrm{C}(\mathrm{n}, \mathrm{p}){ }^{12} \mathrm{~B}\left(1^{\mathrm{st}}\right.$ excited state $)$ & 14.668 & -13.540 & $\mathrm{p}_{1} 2.491$ \\
\hline \multicolumn{4}{|c|}{$\begin{array}{l}{ }^{* 1} \text { The second and higher excited states of }{ }^{12} \mathrm{C} \text { also decay by alpha emission followed by }{ }^{8} \mathrm{Be} \text { breakup into } \\
\text { two alphas, thus contributing to the }{ }^{12} \mathrm{C}\left(\mathrm{n}, \mathrm{n}^{\prime}\right) 3 \alpha \text { channel. } \\
{ }^{* 2} \text { The total charged recoil energy depends on the energy carried away by the scattered neutron leading to a } \\
\text { rather complex continuous neutron spectrum with several peaks, due to different possible paths: via } \\
\text { excited states of }{ }^{12} \mathrm{C} \text {, excited states of }{ }^{9} \mathrm{Be} \text { and }{ }^{5} \mathrm{He} .{ }^{11} \\
{ }^{* 3} \text { Excited states of }{ }^{9} \mathrm{Be} \text { decay also by neutron emission followed by }{ }^{8} \mathrm{Be} \text { breakup into two alphas, thus } \\
\text { contributing to the }{ }^{12} \mathrm{C}\left(\mathrm{n}, \mathrm{n}^{\prime}\right) 3 \alpha \text { channel. }\end{array}$} \\
\hline
\end{tabular}

Further, we attempted to simulate the detector response to monoenergetic fast neutrons on a bare detector and on a detector covered by a HDPE layer of different thicknesses. The effect of the active region thickness and of the incident neutron energy was also considered. The F8 tally has been employed to evaluate detector response; this is the pulse height tally that provides the energy distribution of pulses created in a cell corresponding to the physical detector. It has to be noted that MCNPX has the ability to transport heavy charged particles, but residuals created by nuclear interactions are transported only from the model physics. The use of physics models below $20 \mathrm{MeV}$ is, however, limited and the recoils are mostly stopped due to ionization losses. Therefore, we speculate that the results from alpha and heavy ion transport will have low accuracy.

\section{Results}

The efficiency of neutron-to-proton conversion (calculation details described above) is plotted against HDPE reactive film thickness in Fig. 1. Two curves corresponding to neutron energies of 16.031 and $18.262 \mathrm{MeV}$ show expected maxima, which are about 
$1900 \mu \mathrm{m}$ for $16.031 \mathrm{MeV}$ neutrons and about $2200 \mu \mathrm{m}$ for $18.262 \mathrm{MeV}$ neutrons. The results show that the optimum values of the HDPE thickness are shorter than proton ranges in the film, calculated using the SRIM program, ${ }^{12}$ which are 2.74 and $3.39 \mathrm{~mm}$ for 16 and $18 \mathrm{MeV}$ protons, respectively. The calculated values of the efficiency are relatively low (about $0.25 \%$ ) due to the long free mean path of interacting neutrons in HDPE film in comparison to its thickness and to the short range of the recoil protons in the conversion layer material.

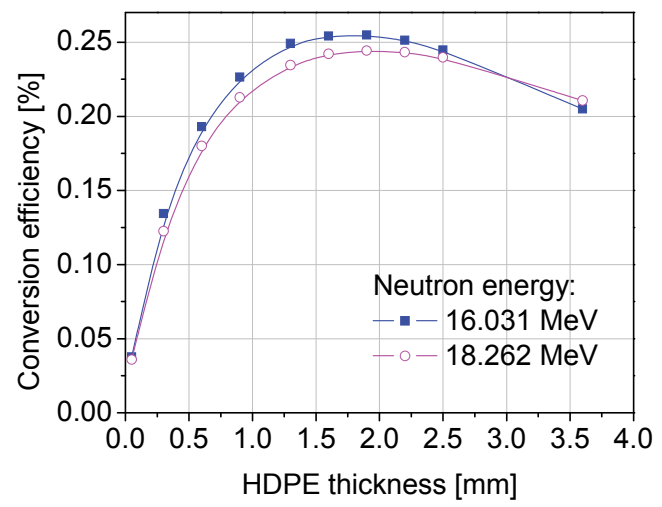

Fig. 1. Conversion efficiency of HDPE reactive layer as a function of its thickness.

As described above, the $\mathrm{SiC}$ detector is able to register neutrons without any conversion layer. The detector response has been simulated using the F8 pulse height tally, which provides the number of pulses depositing energy within the specified energy bins. The F8 tally may have particle combinations, such as F8:a,h,\# (a, alpha particles; h, protons; \#, heavy ions). When secondary particles are indicated on the MODE card, the energy depositions are handled in the regular process of tracking those particles. The F8 tallies of the individual contributing charged particles (alpha particles, protons and heavy ions) together with the overall F8 tally (i.e. F8:a,h,\#), representing a detector response to 16.031 MeV neutrons on bare $\mathrm{SiC}$ material with an active volume thickness of $55 \mu \mathrm{m}$ (corresponding to an applied reverse bias of $-300 \mathrm{~V}$ ), is shown in Fig. 2. The contribution from protons and alpha particles is dominant. As the protons only partially deposit their kinetic energy in the detector active volume, their contribution to the detector response is assigned to lower energies. The contribution from alpha particles has a form of a broad hill in the higher energy region because of their considerably shorter range as compared to lighter protons. Heavy ions contribute to a considerably lower extent in the low energy region. The transport of heavy ions (including alpha particles) and consequently the corresponding deposited energies displayed through the F8 tally is, however, carried out using physics models that do not work reliably in the inspected energy region. Therefore, the contribution from such particles might be different as expected. MCNPX attributes the corresponding energy deposition to the incident neutron, as will be shown later. 


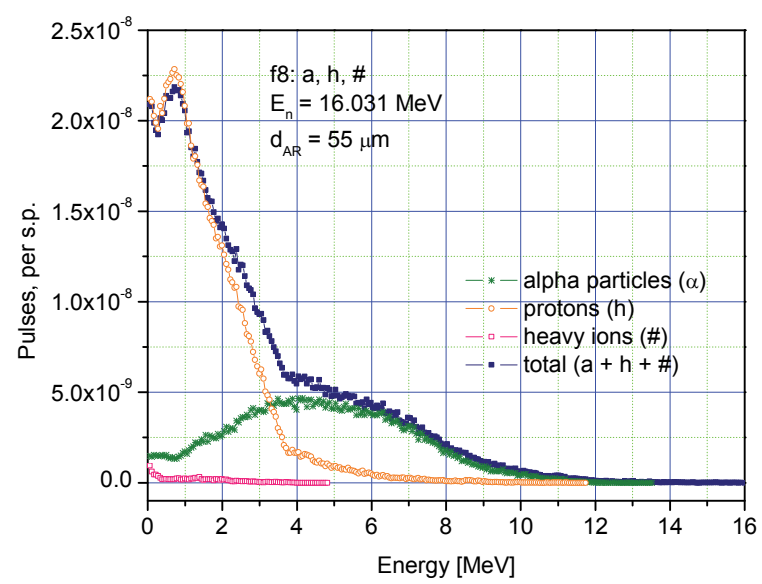

Fig. 2. SiC detector response to $16.031 \mathrm{MeV}$ neutrons. The overall F8:a,h,\# tally as well as F8 tallies for protons, alpha particles and heavy ions separately are shown.

To reveal the energies of secondary charged particles, especially from $(n, \alpha)$ and $(n, p)$ reactions, we have also inspected the F8 tally for neutrons, which stores the total energy deposition in a detector per source particle, including that from secondary particles. The use of the F8:n tally assumes that the fully analogue neutron transport is forced. The resultant pulse height tally is plotted in Fig. 3. In the spectra, many peaks and edges corresponding to nuclear reactions described in Table 1 are revealed (marked in Fig. 3 by arrows), and their position matches well the values listed in the table. However, the pulse heights do not completely match their expected detector response (overestimated in the higher energy region). Similar behavior was already reported and was associated with the fact that the secondary charged particle production is not correlated with the fate of the neutron. ${ }^{13}$ The other aspect affecting this discrepancy in the pulse height underestimates in the lower energy region could be the earlier described limitations of the recoil ion transport.

Detector response simulations using the F8 tally for charged particles were also carried out for different incident neutron energies, i.e. 16.031, 17.543 and $18.262 \mathrm{MeV}$. The effect on the pulse height tally of charged particles exhibited a gentle increase of the spectra in the lower energy field (see Fig. 4a). The same tendency was observed in the measured spectra. Figure $4 \mathrm{~b}$ shows how the change of the active region thickness manifests on the inspected detector response. It can be recognized that the pulse height increases with the thickness of the active region, especially in the low energy region. 


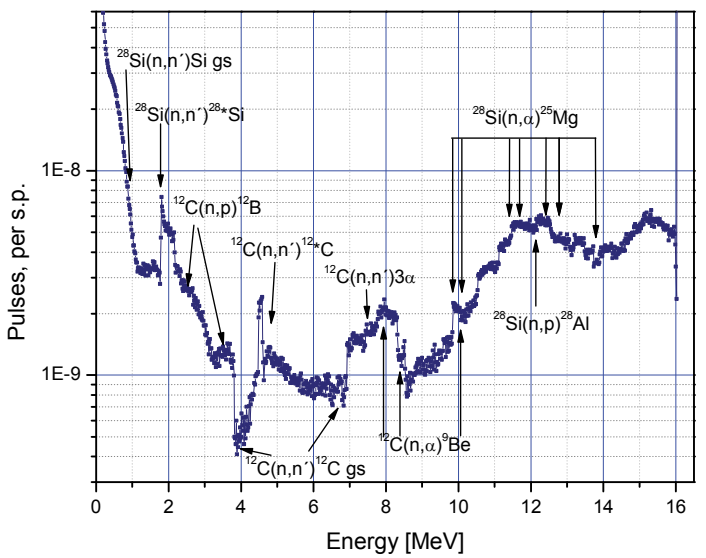

Fig. 3. F8:n,a,h,\# pulse height tally of $\mathrm{SiC}$ detector ( $55 \mu \mathrm{m}$ thick $\mathrm{AR})$ impinged by $16.031 \mathrm{MeV}$ neutrons.
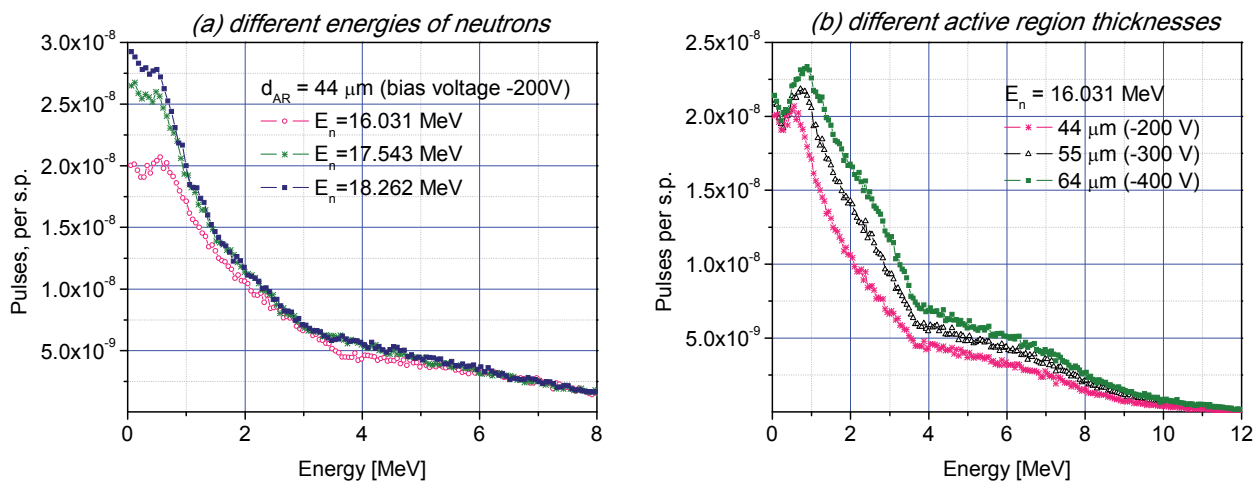

Fig. 4. The effect of neutron energy (a) and AR thickness (b) on the SiC detector response.

Figure 5 displays results from detector response simulations performed for a detector covered with a reactive film of HDPE. The results for a detector covered by a 100, 600 and $1300 \mu \mathrm{m}$ thick conversion layer are plotted against the bare detector response. An observed steep increase of the low energy region pulse heights can be attributed to recoil protons created in the reactive film and entering the active region of the detector. Although the recoil protons can take the whole neutron energy in the process of elastic scattering in HDPE, they deposit only a part of it in the depleted area of the detector due to its small thickness compared to the proton range in $\mathrm{SiC}$. Hence, pulses from protons are present in the lower energy part of the simulated detector response. The contribution from this area increases with rising thickness of the conversion film as the number of protons created and able to escape the layer increases. The same tendency was observed 


\section{K. Sedlačková et al.}

in the measured detector response to monoenergetic fast neutrons. The increased pulse heights in lower energy channels with rising thickness of HDPE film is displayed in Fig. 6 for $16.031 \mathrm{MeV}$ neutrons registered by a SiC detector at a bias of $-200 \mathrm{~V}$.

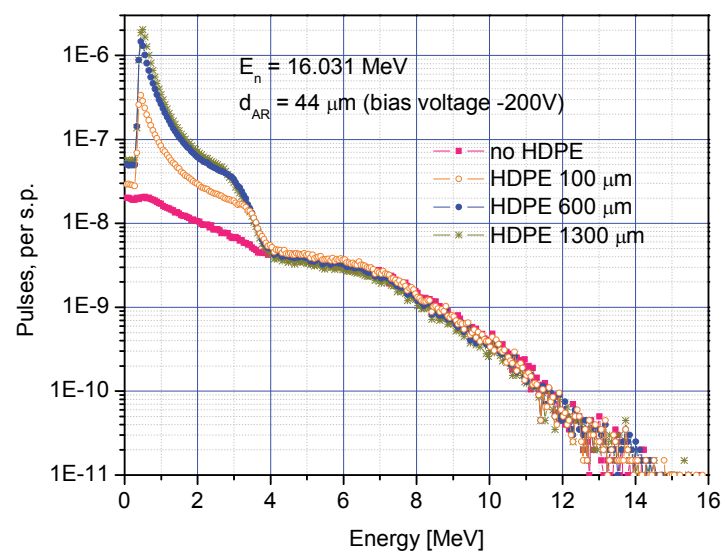

Fig. 5. Detector response simulation for a SiC detector covered with HDPE conversion layer of different thicknesses.

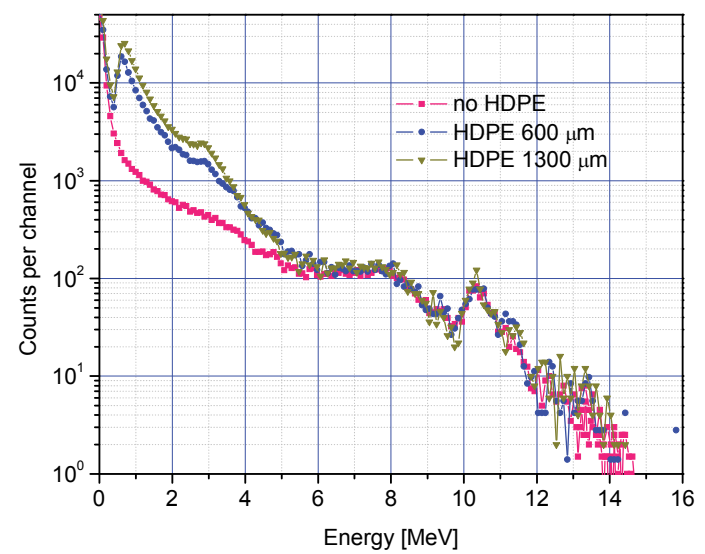

Fig. 6. Measured detector response to $16.031 \mathrm{MeV}$ neutrons at $-200 \mathrm{~V}$ bias.

\section{Conclusion}

The response of a $\mathrm{SiC}$ semiconductor detector to monoenergetic fast neutrons from the D-T nuclear reaction has been simulated using MCNPX. First, the optimal thickness of the HDPE reactive layer was determined and found to lie between 1.9 and $2.2 \mathrm{~mm}$, according to the energy of impinging neutrons (16.031 and $18.262 \mathrm{MeV}$, respectively). Further, the effect of a conversion layer on detector response was studied and spectra with and without a HDPE conversion layer were compared. A pronounced increase in the 
pulse heights was revealed in the lower energy region of the spectra (up to $4 \mathrm{MeV}$ ) where recoil protons represent the most important contribution to the whole energy deposited. The spectra obtained from measurements showed the same tendency. The transport of alpha particles and recoiled ions using MCNPX showed some limitations, and therefore, satisfactory agreement with measurement was not found concerning contributions from these charged particles to the resultant detector response. Positions of the expected peaks from $(n, p)$ and $(n, \alpha)$ reactions can be, however, obtained using the F8 tally for neutrons.

\section{Acknowledgments}

This work was financially supported by a grant of the Slovak Research and Development Agency No. APVV-0321-11, by the Scientific Grant Agency of the Ministry of Education of the Slovak Republic and the Slovak Academy of Sciences No. VEGA 2/0062/13 and by the Ministry of Education, Youth and Sports of the Czech Republic in frame of the research funding grant MSMT No. LM2011030 "VdG Research Infrastructure."

\section{References}

1. F. Franceschini and F. H. Ruddy, Silicon Carbide Neutron Detectors (Chapter 13), Properties and Applications of Silicon Carbide, Prof. Rosario Gerhardt (Ed.), InTech, p. 536, Available from: http://cdn.intechopen.com/pdfs/15095.pdf, (2011).

2. F. H. Ruddy et al., IEEE Transactions on Nuclear Science 53, 3 (2006).

3. F. Moscatelli, A. Scorzoni, A. Poggi and R. Nipoti, Nucl. Instr. Meth. Phys. Res. A 583, 173 (2007).

4. F. H. Ruddy and J. G. Seidel, Nucl. Instr. Meth. Phys. Res. B 263, 163 (2007).

5. P. J. Sellin and J. Vaitkus, Nucl. Instr. Meth. Phys. Res. A 557, 479 (2006).

6. F. Moscatelli, Nucl. Instr. Meth. Phys. Res. A 583, 157 (2007).

7. M. Ivanov, N.B. Strokan and A. A. Lebedev, Nucl. Instr. Meth. Phys. Res. A 597, 203 (2008).

8. D. B. Pelowitz (Ed.), MCNPX Users Manual, Version 2.7.0, Los Alamos National Laboratory Report LA-CP-11-00438, April 2011, pp.1-645.

9. K. Sedlačková et al., Journal of Instrumentation 9, C05016 (2014).

10. P. Selin, Development of semiconductor gamma ray and neutron detectors for security applications, presentation. Available online: https://www.stfc.ac.uk/resources/PDF/PaulSellinpresentation.pdf.

11. K. Kondo et al., Journal of Nuclear Science and Technology 45, 103 (2015).

12. J. F. Ziegler and J. P. Biersack: The Stopping and Range of Ions in Matter, SRIM-2013. www.srim.org, Annapolis, MD (2013).

13. N. Hawkes, Issues with the use of neutron transport codes. Available online: http://www.npl.co.uk/ionising-radiation/issues-with-the-use-of-neutron-transport-codes. 